\section{Lamin-A/C variants found in patients with cardiac conduction disease reduce sodium currents}

\author{
Michael A. Olaopa, ${ }^{1}$ \\ Katherine G. Spoonamore, ${ }^{1}$ \\ Deepak Bhakta, ${ }^{1}$ Zhenhui Chen, ${ }^{1}$ \\ Patricia B.S. Celestino-Soper, ${ }^{2}$ \\ Peng-Sheng Chen, ${ }^{1}$ Tomohiko Ai, ${ }^{1,3}$ \\ Matteo Vatta ${ }^{1,2}$
}

${ }^{1}$ Krannert Institute of Cardiology and

Division of Cardiology, Department of

Medicine, Indiana University School of

Medicine, Indianapolis, IN, USA;

${ }^{2}$ Department of Medical and Molecular

Genetics, Indiana University School of

Medicine, Indianapolis, IN, USA;

${ }^{3}$ Division of Molecular Pathogenesis,

Medical Research Institute, Tokyo

Medical and Dental University, Tokyo, Japan

\begin{abstract}
Variants in the LMNA gene, which encodes Lamin-A/C, have been commonly associated with cardiac conduction system diseases usually accompanying cardiomyopathy. We have seen two unrelated patients who presented with atrioventricular block (AVB) with or without cardiomyopathy. Genetic testing identified the $L M N A$ missense variant c. $1634 \mathrm{G}>\mathrm{A}$ (p.R545H) and the single nucleotide deletion c.859delG (p.A287Lfs*193). The deletion leads to a shift in the reading frame and subsequent protein truncation. Since impaired $\mathrm{Na}_{\mathrm{v}} 1.5$ function has been reported to cause AVB, we sought to investigate the effects of abnormal Lamins on $\mathrm{Na}_{\mathrm{v}} 1.5$ in HEK-293 cells using patch-clamp methods. Patch-clamp studies showed that p.R545H decreased the peak $\mathrm{I}_{\mathrm{Na}}$ by approximately $70 \%$. The voltage-dependency of steady state inactivation was rightward shifted in the cells transfected with p.R545H. The p.A287Lfs*193 also decreased the peak $\mathrm{I}_{\mathrm{Na}}$ by approximately $62 \%$. The voltagedependency of steady state inactivation was rightward shifted in the cells transfected with p.A287Lfs*193. Variants of the LMNA gene caused significant reduction of the peak $\mathrm{I}_{\mathrm{Na}}$ in HEK-293 cells, which may account for the patients' AVB.
\end{abstract}

\section{Introduction}

The nuclear lamina is a proteinaceous layer underlying the inner membrane of the nucleus. Lamin-A/C, which is a nuclear intermediate filament protein, primarily interacts with cytoskeletal structures to provide cell adhesion functions. ${ }^{1,2}$ This interaction with cytoskeletal structures, such as $\alpha$-actinin, desmin, and other linker proteins, may provide the machinery by which modulation of sarcolemma proteins, such as Nav1.5, might occur. ${ }^{3,4}$ Laminopathies are a series of genetic disorders, characterized by cardiac and skeletal anomalies. LMNA variants have been linked with these disorders, which include over 12 clinically heterogeneous syndromes. ${ }^{1}$ Within the heart, LMNA variants are the major cause of primary dilated cardiomyopathy (DCM) with conduction system disease, including atrioventricular block (AVB). ${ }^{5}$ Majority of the patients require pacemaker or implantable cardioverter-defibrillator (ICD) therapy to prevent sudden cardiac death. ${ }^{6}$ However, due to a wide range of clinical presentations, the mechanisms of disease development are still poorly understood. It has been reported that the dysfunction of cardiac sodium channel $\left(\mathrm{Na}_{\mathrm{v}} 1.5\right)$ due to the genetic abnormalities of $S C N 5 A$, the gene encoding $\mathrm{Na}_{\mathrm{v}} 1.5$, or its modulator proteins can cause cardiac conduction diseases. ${ }^{7} \mathrm{We}$ have identified two $L M N A$ variants from patients presented with cardiac conduction defects AVB with and without DCM. We hypothesized that Lamin-A/C mutation may cause AVB by reducing $\mathrm{Na}_{\mathrm{v}} 1.5$. To test that hypothesis, we performed patch-clamp studies in human embryonic kidney (HEK293) cells expressing these variants. We showed that both variants significantly decreased the peak sodium current $\left(\mathrm{I}_{\mathrm{Na}}\right)$ by approximately $60-70 \%$ and affected the voltage dependency of $\mathrm{Na}_{\mathrm{v}} 1.5$. These findings suggest that the reduction of $\mathrm{I}_{\mathrm{Na}}$ by the $L M N A$ variants might contribute to the patients' electrocardiographic phenotypes.

\section{Materials and Methods}

All patients and their family members were given oral explanations and written informed consents for the participation of this study. The study protocols were designed following the Helsinki declaration, and were approved by the institutional review board of Indiana University.

\section{Patient characteristics}

Case 1: The patient was a 34-year old Caucasian male. He was diagnosed with advanced AVB (3:1 AV conduction) with left anterior hemi block and right bundle branch block, and underwent pacemaker
Correspondence: Matteo Vatta, Department of Medical and Molecular Genetics, Indiana University School of Medicine, Indianapolis, IN, USA. E-mail: mvatta@iu.edu

Tomohiko Ai, Krannert Institute of Cardiology, Department of Medicine, Indiana University School of Medicine, Indianapolis, IN, USA; Division of Molecular Pathogenesis, Medical Research Institute, Tokyo Medical and Dental University, Tokyo, Japan. E-mail: ait@iu.edu.

Key words: Lamin A/C; atrioventricular block; cardiomyopathy; sodium channel.

Acknowledgements: the authors are grateful to the patients and their family members who participated in this study. We are also grateful to Jian Tan for his technical assistance.

Funding: this study was supported in part by 1P01 HL78931, R01 HL71140, R42DA043391 (PSC) the Methodist Research Institute, Showalter Cardiovascular Research Fund (TA), the Indiana University HealthIndiana University School of Medicine Strategic Research Initiative (PSC, TA, MV and PBSC-S), and by the Dr. Charles Fisch Cardiovascular Research Award endowed by Dr. Suzanne B. Knoebel (MAO).

Contributions: TA and MV contributed equally, conceptualization and study design; MAO, KGS, PBSC-S, ZC, MV, TA data acquisition and analyses; MAO, KGS, PBSC-S, MV and TA drafting the manuscript; DB, PSC, ZC, TA and MV significantly reviewing the manuscript.

Conflict of interests: the authors declare no potential conflict of interests.

Received for publication: 7 October 2017 Revision received: 12 December 2017.

Accepted for publication: 30 December 2017.

This work is licensed under a Creative Commons Attribution NonCommercial 4.0 License (CC BY-NC 4.0).

(C) Copyright M.A. Olaopa et al., 2018

Licensee PAGEPress, Italy

Cardiogenetics 2018; 8:7127

doi:10.4081/cardiogenetics.2018.7127

implantation (Figure 1A). However, he had no symptoms or echocardiogram findings indicating heart failure. Interestingly, his son presented with prenatal bradycardia and was diagnosed with second degree AVB at age two. Figure 1B shows the pedigree. There is no known family history of heart failure or sudden cardiac death.

Case 2: The patient was a 34-year old Caucasian male with sudden cardiac arrest, DCM, and family history of DCM and arrhythmia. The patient had conduction 
system disease, including first degree AVB and incomplete left bundle branch block, manifested by progressive PR prolongation and slightly increased QRS duration (Figure 2A). An electrocardiogram (ECG) taken two years later showed further lengthening of PR interval (Figure 2B). Although the patient underwent ICD implantation, his condition continued to deteriorate. He subsequently underwent orthotopic heart transplantation. Figure 2C shows the pedigree.

\section{Genetic tests}

The DNA samples extraction and sequencing procedures were performed by a National Clinical Laboratory using a next generation sequencing panel of 38 genes including: $K C N Q 1, K C N H 2$, $S C N 5 A$, $A N K 2$, KCNE1, KCNE2, KCNJ2, CAV3, RYR2, CASQ2, LMNA, LDB3/ZASP, TNNT2, DES, SGCD, PLN, ACTC1, MYH7, TPM1, TNNI3, TAZ, TTR, MYBPC3, LAMP2, MTTK, MTTL1, MTTL2, MTTQ, MTTH, MTTD, MTTI, MTTM, MTTS1, MTTS2, MTND1, MTND5, MTND6 (for Case1); ACTC1, ACTN2, ANKRD1, CSRP3, DES, EMD, LAMP2, LMNA, MTND1, MTND5, MTND6, MTTD, MTTH, MTTI, MTTK, MTTL1, MTTL2, MTTM, MTTQ, MTTS1, MTTS2, MYBPC3, MYH7, NEXN, PLN, RBM20, SCN5A, SGCD, TAZ, TCAP, TNNC1, TNNI3, TNNT2, TPMI, TTN, TTR, VCL, ZASP (for Case 2).

\section{Construction of the plasmids and cell preparation}

The cDNA of LMNA was purchased from OriGene Technologies (Rockville, MD). The cDNA of WT-LMNA (the ORF based upon NM_005572) was inserted in the pCMV6-AC-GFP vector. Mutant-LMNA constructs (p.R545H and p.A287Lfs*193) were made using site-direct mutagenesis kit (Qiagen). Sequences of the all plasmids were confirmed with direct sequencing.

\section{Cell culture and transfection}

HEK-293 cells were grown and maintained in IMDM (Life Technologies), supplemented with $10 \%$ fetal bovine serum (Sigma Aldrich). It has been reported that the splice variant lacking glutamine at position 1077 (Q1077del) reaches $65 \%$ of the SCN5A transcript in human heart. ${ }^{8}$ Therefore, cells were transiently transfected with $S C N 5 A$ Q1077del and WT or mutant LMNAs for 48 hours using Effectene reagent (Qiagen) according to the manufacturer's instructions in 6-well plates. Each well was transfected with no more than $2 \mu \mathrm{g}$ combined DNA. Cells were harvested using trypsin-EDTA (Life Technologies) for 1-2 mins, and transferred to the patch chamber for recording.

\section{Patch-clamp experiments}

Whole cell mode of the voltage-clamp technique was used in this study as described elsewhere. ${ }^{9}$ Briefly, whole-cell configuration was made in Tyrode's solution. Pipette resistances were 1.5-3 M $\Omega$. After achieving a giga seal, the test-pulse current was nulled by adjusting the pipette capacitance compensator with both fast and slow components. After break-in, the whole-cell charging transient was nulled by adjusting whole cell capacitance and series resistance. Voltage control protocols were generated with Axopatch 200B amplifier/Digidata 1440A acquisition system using pCLAMP10 software (Molecular Devices/Axon, Sunnyvale, CA). Whole-cell recording was analyzed using Clampfit 10.2. For measuring
$\mathrm{I}_{\mathrm{Na}}$, we used Tyrode's solution as the bath solution. The pipette solution contained (in $\mathrm{mM}) \mathrm{NaF} 10, \mathrm{CsF} 110, \mathrm{CsCl} 20$, EGTA 10, and HEPES $10(\mathrm{pH} 7.35$ adjusted with $\mathrm{CsOH}$ ). All experiments were carried out at room temperature.

\section{Statistical analysis}

Related-samples Friedman's Two-Way Analysis of Variance by Ranks was conducted. Related-Samples Wilcoxon Signed Rank Test was performed for posthoc analysis. P value less than 0.05 was considered as statistically significant. Statistical analyses were performed using SPSS (IBM, Chicago, IL, USA, version 21). Data in text and figures are presented as mean \pm S.E.
(A)

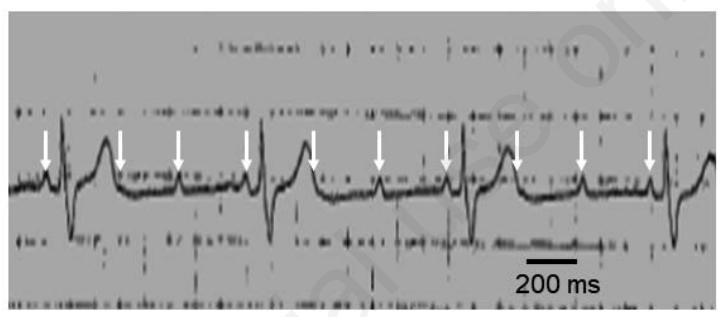

(B)

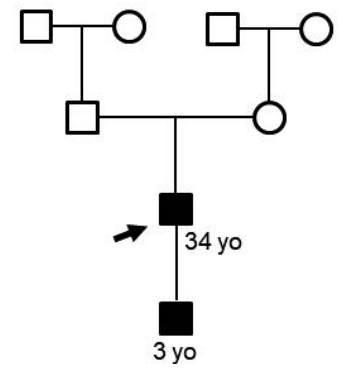

Figure 1. Clinical characteristics of Case 1. A) ECG strips of Case 1. The arrows indicate the timing of $P$ waves. $B$ ) Pedigree of Case 1 . The arrow indicates the proband. Shading indicates individuals with cardiac conduction abnormalities.

(A)

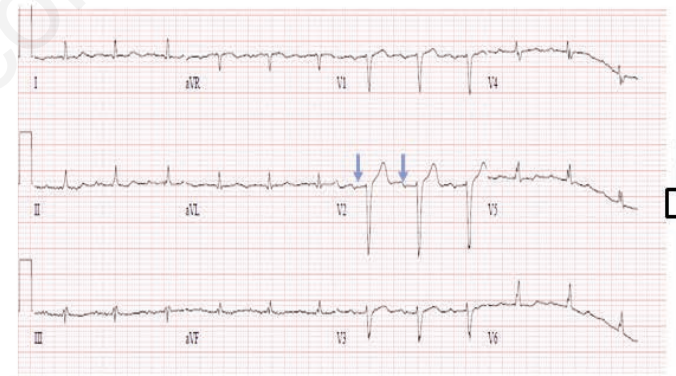

(B)

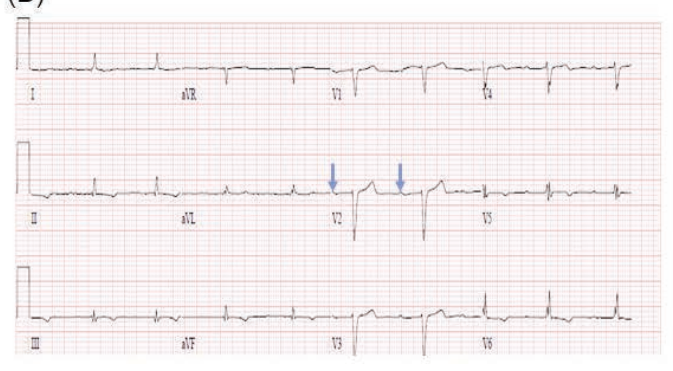

(C)

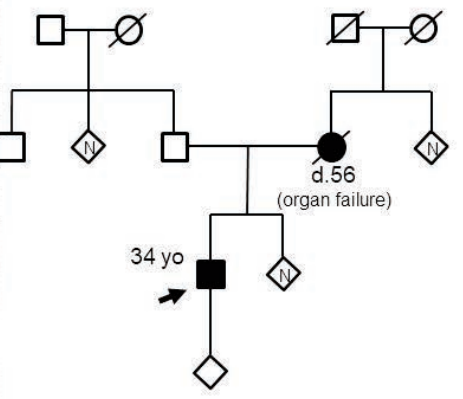

Figure 2. Clinical characteristics of case 2. A) Twelve lead ECG of Case 2. ( $P R=284 \mathrm{~ms}$, $\mathrm{QRS}=112 \mathrm{~ms})$. B) Twelve lead ECG obtained two years later $(P R=422 \mathrm{~ms}, \mathrm{QRS}=104$ $\mathrm{ms}$ ). Note that the PR intervals are markedly prolonged. The arrows indicate the timing of $P$ waves in V2. C) Pedigree of Case 2. The arrow indicates the proband. Shading indicates individuals with cardiomyopathy. 
$-50.42 \pm 0.32 \mathrm{mV}, \mathrm{N}=10$, N.S.).

Next, we examined the effect of p.A287Lfs*193-LMNA on $\mathrm{I}_{\mathrm{Na}}$, and compared the parameters with WT-LMNA. Figure 5A shows the representative superimposed current traces of $\mathrm{I}_{\mathrm{Na}}$ obtained from cells transfected with SCN5A and WT-LMNA or SCN5A and p.A287Lfs*193-LMNA. p.A287Lfs*193-LMNA significantly decreased the peak $\mathrm{I}_{\mathrm{Na}}$ by $62 \%$ compared to the WT- $L M N A$ (WT: $340 \pm 45 \mathrm{pA} / \mathrm{pF}, \mathrm{N}=10$ $v s$ p.A287Lfs*193: $132 \pm 28 \mathrm{pA} / \mathrm{pF}, \mathrm{N}=9$, $\mathrm{P}<0.005)$. Figure $5 \mathrm{~B}$ shows the $\mathrm{I}-\mathrm{V}$ relationships. Figure $5 \mathrm{C}$ shows that the voltage-dependency of the steady-state inactivation was rightward shifted in the cells transfected with p.A287Lfs *193 $\left(V_{h}\right.$ : WT, $\quad-93.20 \pm 0.89 \quad \mathrm{mV}, \quad \mathrm{N}=15 \quad$ vs p.A287Lfs*193, $-90.10 \pm 1.92 \mathrm{mV}, \mathrm{N}=8$, $\mathrm{P}<0.05 ; \quad k$ : WT, $5.62 \pm 0.07 \quad$ vs p.A287Lfs*193, $9.18 \pm 0.24, \quad \mathrm{P}<0.005)$. However, steady state activation was unaffected $\left(V_{h}\right.$ : WT, $-52.38 \pm 0.41 \mathrm{mV}, \mathrm{N}=10$ vs p.A287Lfs $* 193,-50.0 \pm 0.26 \mathrm{mV}, \mathrm{N}=9$, N.S.). Table 1 summarizes these $I_{\mathrm{Na}}$ parameters.

Since impaired $\mathrm{Na}_{\mathrm{v}} 1.5$ function has bee reported to cause $\mathrm{AVB},{ }^{10}$ we sought to investigate whether these abnormal LMNAs can alter channel function. HEK-293 cells were co-transfected with SCN5A and GFPconjugated WT-, p.R545H-, or p.A287Lfs*193-LMNA. $\mathrm{I}_{\mathrm{Na}}$ was measured and compared among the cells transfected with WT or mutant LMNAs using patchclamp methods.

Figure 4A shows representative superimposed current traces obtained from HEK-293 cells transfected with SCN5A alone (top panel), SCN5A and p.R545HLMNA (middle panel), or SCN5A and WT-LMNA (bottom panel). Very interestingly, WT-LMNA drastically increased the peak $\mathrm{I}_{\mathrm{Na}}$ (bottom trace) by approximately three-fold compared to the baseline SCN5A (WT-LMNA: 340 45 $\mathrm{pA} / \mathrm{pF}, \mathrm{N}=10$ vs $S C N 5 A$ only: $135 \pm 21$ $\mathrm{pA} / \mathrm{pF}, \mathrm{N}=7, \mathrm{P}<0.005$ ). Figure $3 \mathrm{~A}$ shows the current-voltage $(\mathrm{I}-\mathrm{V})$ relationships. The voltage-dependency of the steady-state activation and inactivation was also leftward-shifted by WT-LMNA (Figure 3B, Table 1). Figure 4B shows the I-V relationships. p.R545H-LMNA failed to activate peak $\mathrm{I}_{\mathrm{Na}}$ by $70 \%$ compared to the cells transfected with WT-LMNA (WT: $340 \pm 45 \mathrm{pA} / \mathrm{pF}, \mathrm{N}=10$ vs p.R545H: $95 \pm 15$ $\mathrm{pA} / \mathrm{pF}, \mathrm{N}=10, \mathrm{P}<0.005)$. Figure $4 \mathrm{C}$ shows that p.R545H-LMNA shifted the voltagedependency of the steady-state inactivation towards positive compared to WT-LMNA $\left(V_{h}:\right.$ WT, $-93.20 \pm 0.89 \quad \mathrm{mV}, \quad \mathrm{N}=15 \quad v s$ p.R545H, $\quad-87.64 \pm 0.91 \quad \mathrm{mV}, \quad \mathrm{N}=24$, $\mathrm{P}<0.005)$. However, the steady-state activation was unaffected $\left(V_{h}\right.$ : WT, $52.38 \pm 0.41 \mathrm{mV}, \quad \mathrm{N}=10 \quad$ vs $\quad$ p.R545H,

Table 1. Summary of INa parameters.

${ }^{*} \mathrm{P}<0.05$ vs WT; ${ }^{*} \mathrm{P}<0.005$ vs WT.

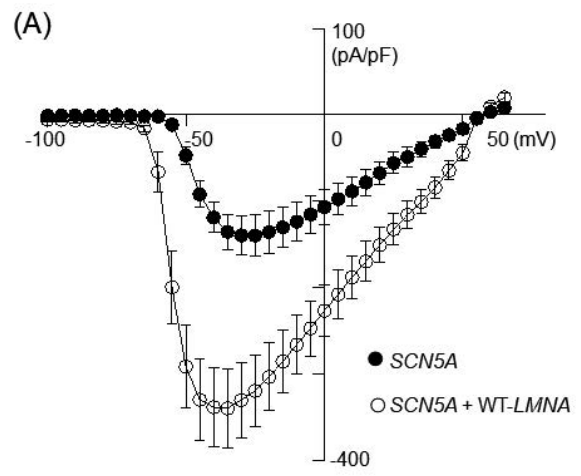
tion obtained from the transfected cells.

\section{Discussion and Conclusions}

In this study, we found two variants in the LMNA gene, p.R545H and p.A287Lfs*193, in two patients who showed AVB. The latter patient also had cardiomyopathy. Our patch-clamp experiments using HEK-293 cells showed that the peak $\mathrm{I}_{\mathrm{Na}}$ was reduced when these two variants were co-expressed with SCN5A. The data indicate that these $L M N A$ variants may fail to activate $\mathrm{Na}_{\mathrm{v}} 1.5$ properly, resulting in the reduction of $\mathrm{I}_{\mathrm{Na}}$ compared to cells transfected with WT-LMNA. Since the magnitudes of $\mathrm{I}_{\mathrm{Na}}$ reduction was greater than $50 \%$, this might account for the patients' ECG phenotypes.

Several previous studies, including ours, demonstrated that the function of $\mathrm{Na}_{\mathrm{v}} 1.5 \mathrm{can}$ be modulated via various cytoskeletal proteins such as actin, ${ }^{3} \alpha$-actinin- $2,{ }^{11}$ syntrophin and dystrophin complex, ${ }^{12}$ and ZASP/Telethonin cytoskeletal complex. ${ }^{4}$ The proposed underlying mechanisms of these functional modifications of $\mathrm{Na}_{\mathrm{v}} 1.5$ by the cytoskeletal proteins are either alteration of protein expression or modification of ion channel kinetics. However, the exact mechanisms remain elusive. On the contrary,

$\begin{array}{lcccc} & \text { SCN5A only } & \text { WT } & \text { p.R545H } & \text { p.A287Lfs* } 193 \\ & \mathrm{~N}=7 & \mathrm{~N}=10 & \mathrm{~N}=10 & \mathrm{~N}=9 \\ \text { Peak } \mathrm{I}_{\mathrm{Na}}(\mathrm{pA} / \mathrm{pF}) & 135 \pm 21^{* *} & 340 \pm 45 & 95 \pm 15^{* *} & 132 \pm 28^{* *} \\ & \mathrm{~N}=7 & \mathrm{~N}=10 & \mathrm{~N}=10 & \mathrm{~N}=9 \\ \text { Steady-state activation } & -48.20 \pm 0.55^{*} & -52.38 \pm 0.41 & -50.42 \pm 0.32 & -50.0 \pm 0.26 \\ V_{h}(\mathrm{mV}) & 5.97 \pm 0.40 & 5.21 \pm 0.36 & 4.88 \pm 0.29 & 6.82 \pm 0.23 \\ \mathrm{k} & \mathrm{N}=8 & \mathrm{~N}=15 & \mathrm{~N}=24 & \mathrm{~N}=8 \\ \text { Steady-state inactivation } & -82.10 \pm 0.95^{* *} & -93.20 \pm 0.89 & -87.64 \pm 0.91^{* *} & -90.10 \pm 1.92^{*} \\ V_{h}(\mathrm{mV}) & 4.95 \pm 0.24 & 5.62 \pm 0.07 & 6.54 \pm 0.08 & 9.18 \pm 0.24^{* *} \\ k\end{array}$

(B)

Figure 3. Patch clamp analyses on HEK-293 cells transfected with SCN5A and WT$L M N A$. A) I-V relationship obtained from the cells transfected with SCN5A alone or SCN5A + WT-LMNA. B) Voltage-dependency in the steady-state activation and inactiva-

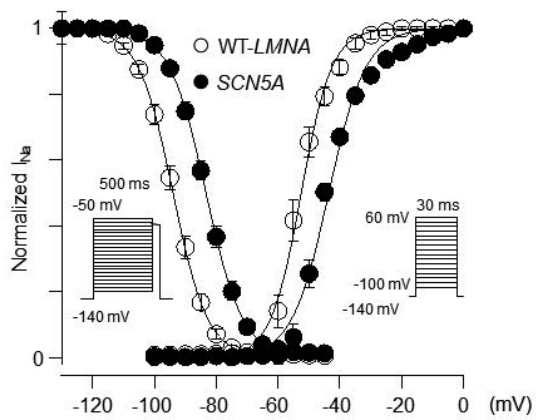


it has been reported that the structural proteins of nucleus and nucleoskeleton may link with cytoskeletal proteins, called linker of nucleoskeleton and cytoskeleton (LINC) complex. For example, nesprin $1 \alpha$ is essential to determine the position of nucleus in muscle cells and cytoskeletal muscle functions. ${ }^{13}$ Nesprins interact with desmins and microtubles that play important role in the function of muscles. ${ }^{14,15}$ Taken these together, it is reasonable to speculate that the structural changes of Lamin-A/C, caused by genetic variants, can affect the function of $\mathrm{Na}_{\mathrm{v}}$ 1.5.

In our cases, the first patient showed advanced AVB without any heart failure symptoms, and he has no family history of cardiomyopathy. On the contrary, the second patient showed both AVB and cardiomyopathy. The patient's mother showed obvious DCM, and her brother and father had symptoms suggesting cardiomyopathy. The question remains whether or not the ECG phenotypes in these two individuals are caused by the same underlying mechanism. As we reported in our previous studies, patients with abnormal cytoskeletal structures may show their arrhythmia phenotypes preceding their cardiomyopathy phenotypes. ${ }^{4,16}$ Since crystallography studies were not available, we do not know the actual structural abnormalities caused by these two variants of LMNA. The first patient's LMNA abnormality is a single nucleotide variant (SNV) in exon 10, and the second patient's $L M N A$ abnormality in exon 5 is a single nucleotide deletion, leading to a frameshift and premature termination of $L M N A$. Since WT- $L M N A$ activated the $\mathrm{I}_{\mathrm{Na}}$, the mutated proteins might have failed to activate the $\mathrm{I}_{\mathrm{Na}}$ properly (Figure 4A-B; Figure 5A-B). In addition, p.A287Lfs*193-LMNA might be degraded by nonsense-mediated mRNA decay in actual human hearts as well. ${ }^{17}$ The latter genotype is known to be associated with more severe clinical manifestations of the disease. ${ }^{18}$

Interestingly, p.R545H-LMNA was found in Dunnigan-type 2 familial partial lipodystrophy (FPLD2). ${ }^{19}$ However, in the clinical record we obtained, no evidence that a FPLD2 phenotype was documented. The clinical manifestation of this variant might be similar to what occurs in Anderson-Tawil syndrome (Long-QT 7). ${ }^{20}$ Although patients with pathogenic variants in the causative gene of LQT7, KCNJ2, can present with various dysmorphic features, periodic paralysis, and QT-prolongation, the clinical phenotypes vary a lot depending upon individual and age. Isolated LQTS was also observed. Therefore, long-term close follow up is warranted for our patients.
(A)

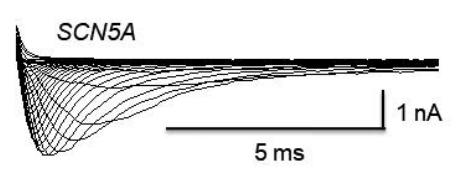

SCN5A+p.R545H-LMNA
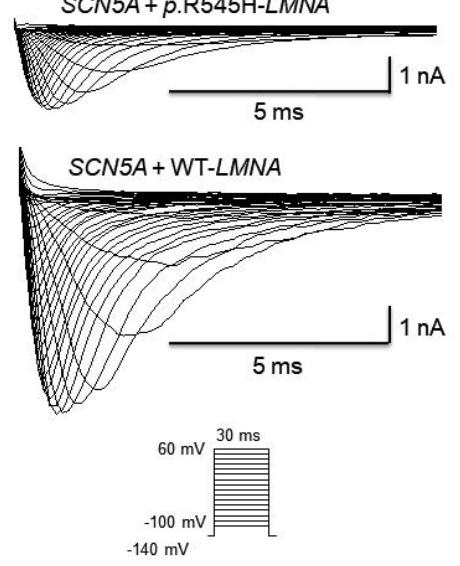

(B)
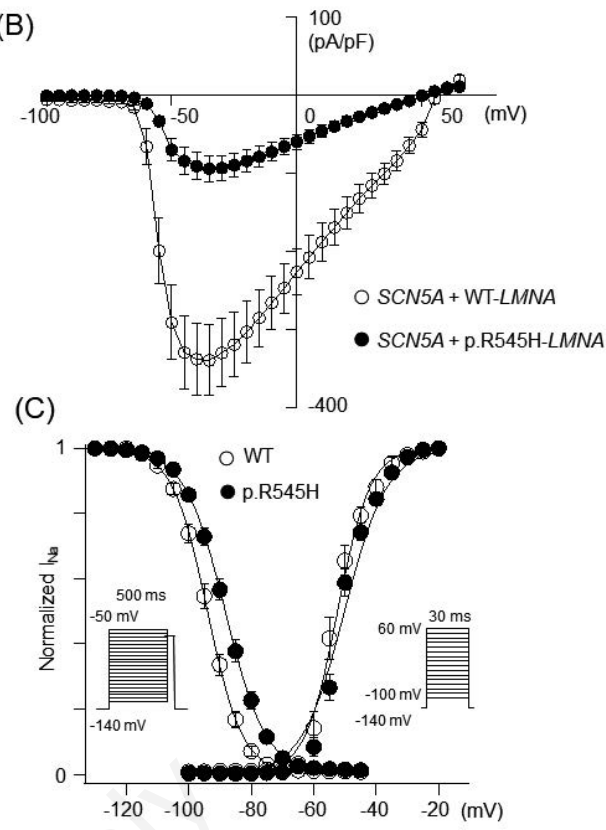

Figure 4. Patch clamp analyses on HEK-293 cells transfected with SCN5A and WT- or p.R545H-LMNA. A) Representative current traces obtained from the cells co-transfected with $S C N 5 A$ only, $S C N 5 A$ with WT- or p. $\mathrm{R} 545 \mathrm{H}-L M N A$ plasmids. The $\mathrm{I}_{\mathrm{Na}}$ was induced by repetitive step pulses $(30 \mathrm{~ms}$, from $-100 \mathrm{mv}$ to $60 \mathrm{mV}$ with a holding potential of -140 $\mathrm{mV})$. B) I-V relationship. C) Voltage-dependency in the steady-state activation and inactivation obtained from the cells transfected with $S C N 5 A$ with WT-LMNA or p.R545H$L M N A$. Conductance $\mathrm{G}(\mathrm{V})$ was calculated by the equation: $\mathrm{G}(\mathrm{V})=\mathrm{I} /\left(\mathrm{V}_{\mathrm{m}}-\mathrm{E}_{\mathrm{rev}}\right)$, where $\mathrm{I}$ is the peak currents, $\mathrm{E}_{\text {rev }}$ is the measured reversal potential, and $\mathrm{Vm}$ is the membrane potential. The normalized peak conductance was plotted as a function of membrane potentials. Steady-state inactivation was estimated by pre-pulse protocols $(500 \mathrm{~ms})$ from a holding potential of $-140 \mathrm{mV}$. Steady state activation and inactivation were fitted with the Boltzmann equation: $y=\left[1+\exp \left(\left(V_{m}-V_{b}\right) / k\right)\right]^{-1}$, where y represents variables; $V_{b}$, midpoint; $k$, slope factor; $V_{m}$, membrane potential. Data were represented as mean \pm S.E.

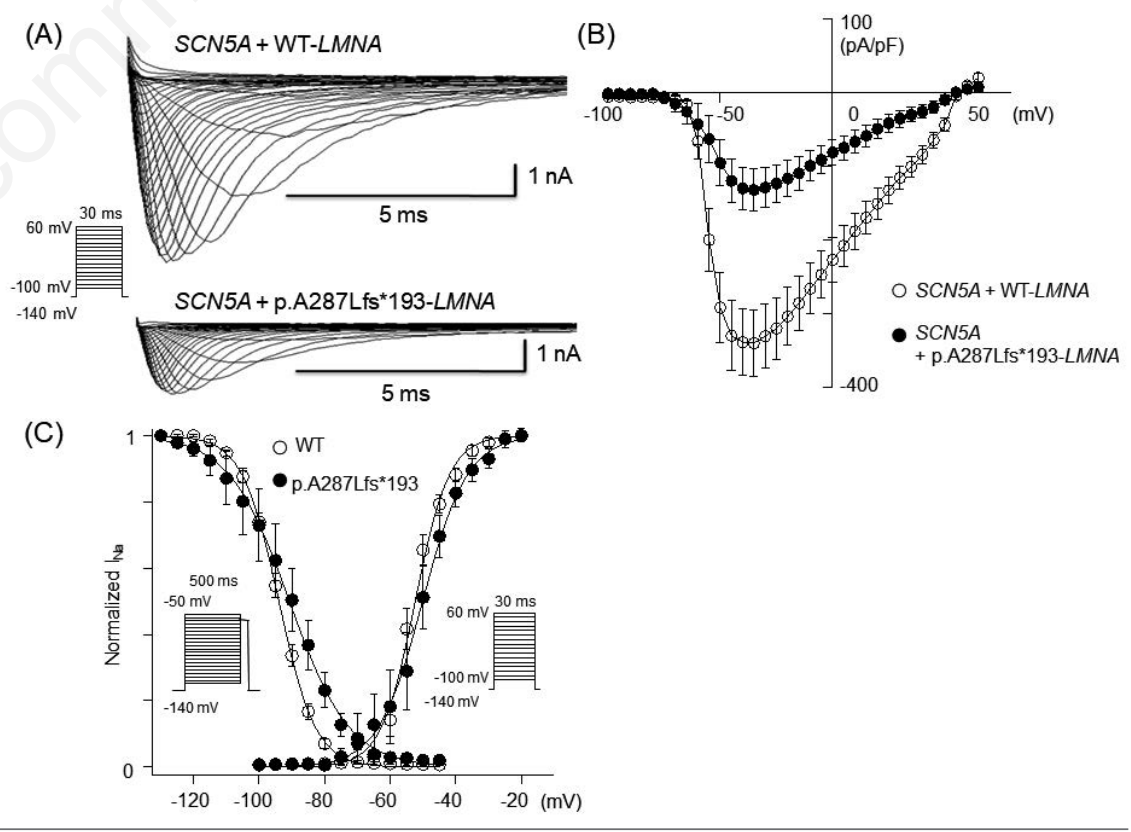

Figure 5. Patch clamp analyses on HEK-293 cells transfected with WT- or p.A287Lfs*193-LMNA. A) Representative current traces obtained from the cells transfected with various plasmids. $I_{\mathrm{Na}}$ was induced by repetitive step pulses $(30 \mathrm{~ms}$, from $-100 \mathrm{mv}$ to $60 \mathrm{mV}$ with a holding potential of $-140 \mathrm{mV}$ ). B) I-V relationship obtained from the cells transfected with various combinations of the plasmids. C) Voltage-dependency in the steady-state activation and inactivation obtained from the cells transfected with $S C N 5 A$ with WT-LMNA or p.A287Lfs*193-LMNA. 
There are several limitations in our study. Since biopsies and detailed histological studies of the patients' hearts were not performed, we cannot exclude a possibility that the fibrosis associated with cardiomyopathy was the main cause of the conduction disturbances as mentioned above. Since detailed information regarding studies for ischemic heart diseases was not provided, we cannot rule out the ischemic causes for their ECG phenotypes. However, there were no underlying factors known to increase the risk of ischemic heart disease and any symptoms suggesting ischemic heart diseases. In addition, the patients' ages are relatively young (3 y.o. and 34 y.o.), and ECG did not show any apparent ischemic changes. Thus, we think ischemic causes are less likely. In our study, a heterologous system using HEK-293 cells was employed to investigate the effects of WT and mutant $L M N A s$ on the function of $\mathrm{hNa}_{\mathrm{v}} 1.5$. Since the cytoskeletal structure, signal transductions, and protein expression profiles of HEK-293 cells are different from cardiomyocytes, we do not know if the reduction of $\mathrm{I}_{\mathrm{Na}}$ by $L M N A$ variants actually took place in the patients' hearts. This limitation applies to all HEK293 studies, which might be improved if we use different assay models such as animal models and iPSC-derived cardiomyocytes. However, these are beyond the scope of current study. In addition, since the detailed study in regard to protein-protein interaction and single channel recording of $\mathrm{Na}_{\mathrm{v}} 1.5$ were not yet performed, we could not reveal exact underlying mechanisms of how LMNA can affect $\mathrm{I}_{\mathrm{Na}}$. Therefore, further studies are warranted in the future.

In conclusion, disease-causing LMNA variants may reduce peak $\mathrm{I}_{\mathrm{Na}}$ when coexpressed with SCN5A in HEK-293 cells. These findings suggest that reduced peak $\mathrm{I}_{\mathrm{Na}}$ may play a role in the development of AVB in these two families.

\section{References}

1. Bertrand AT, Chikhaoui K, Yaou RB, Bonne G. Clinical and genetic heterogeneity in laminopathies. Biochem Soc Trans 2011;39:1687-92.

2. Houben F, De Vos WH, Krapels IP, et al. Cytoplasmic localization of PML particles in laminopathies. Histochem Cell Biol 2013;139:119-34.

3. Undrovinas AI, Shander GS, Makielski JC. Cytoskeleton modulates gating of voltage-dependent sodium channel in heart. Am J Physiol 1995;269:H203-14.

4. Li Z, Ai T, Samani K, et al. A ZASP missense mutation, S196L, leads to cytoskeletal and electrical abnormalities in a mouse model of cardiomyopathy. Circ Arrhythm Electrophysiol 2010;3:646-56.

5. Kumar S, Baldinger SH, Gandjbakhch $\mathrm{E}$, et al. Long-term arrhythmic and nonarrhythmic outcomes of lamin $\mathrm{A} / \mathrm{C}$ mutation carriers. J Am Coll Cardiol 2016;68:2299-307.

6. Anselme F, Moubarak G, Savoure A, et al. Implantable cardioverter-defibrillators in lamin $\mathrm{A} / \mathrm{C}$ mutation carriers with cardiac conduction disorders. Heart Rhythm 2013;10:1492-8.

7. Wang DW, Viswanathan PC, Balser JR, et al. Clinical, genetic, and biophysical characterization of SCN5A mutations associated with atrioventricular conduction block. Circulation 2002;105:341-6.

8. Makielski JC, Ye B, Valdivia CR, et al. A ubiquitous splice variant and a common polymorphism affect heterologous expression of recombinant human SCN5A heart sodium channels. Circ Res 2003;93:821-8.

9. $\mathrm{Yu}$ CC, Ai T, Weiss JN, Chen PS. Apamin does not inhibit human cardiac $\mathrm{Na}+$ current, L-type $\mathrm{Ca} 2+$ current or other major $\mathrm{K}+$ currents. PLoS One 2014;9:e96691.

10. Barra SN, Providencia R, Paiva L, et al. A review on advanced atrioventricular block in young or middle-aged adults.
Pacing Clin Electrophysiol 2012;35: 1395-405.

11. Ziane R, Huang H, Moghadaszadeh B, et al. Cell membrane expression of cardiac sodium channel $\mathrm{Na}(\mathrm{v}) 1.5$ is modulated by alpha-actinin-2 interaction. Biochemistry 2010;49:166-78.

12. Gavillet B, Rougier JS, Domenighetti AA, et al. Cardiac sodium channel Nav1.5 is regulated by a multiprotein complex composed of syntrophins and dystrophin. Circ Res 2006;99:407-14.

13. Stroud MJ, Banerjee I, Veevers J, Chen J. Linker of nucleoskeleton and cytoskeleton complex proteins in cardiac structure, function, and disease. Circ Res 2014;114:538-48.

14. Chapman MA, Zhang J, Banerjee I, et al. Disruption of both nesprin 1 and desmin results in nuclear anchorage defects and fibrosis in skeletal muscle. Hum Mol Genet 2014;23:5879-92.

15. Stroud MJ, Feng W, Zhang J, et al. Nesprin 1alpha2 is essential for mouse postnatal viability and nuclear positioning in skeletal muscle. J Cell Biol 2017; 216:1915-24.

16. Xi Y, Ai T, De Lange E, et al. Loss of function of hNav1.5 by a ZASP1 mutation associated with intraventricular conduction disturbances in left ventricular noncompaction. Circ Arrhythm Electrophysiol 2012;5:1017-26.

17. Brogna S, Wen J. Nonsense-mediated mRNA decay (NMD) mechanisms. Nat Struct Mol Biol 2009;16:107-13.

18. MacLeod HM, Culley MR, Huber JM, et al. Lamin $\mathrm{A} / \mathrm{C}$ truncation in dilated cardiomyopathy with conduction disease. BMC Med Genet 2003;4:4.

19. Chan D, McIntyre AD, Hegele RA, Don-Wauchope AC. Familial partial lipodystrophy presenting as metabolic syndrome. J Clin Lipidol 2016;10: 1488-91.

20. Ai T, Fujiwara Y, Tsuji K, et al. Novel KCNJ2 mutation in familial periodic paralysis with ventricular dysrhythmia. Circulation 2002;105:2592-4. 